\title{
Angiogenesis in Diabetes. Unraveling the Angiogenic Paradox
}

\author{
Raquel Soares*
}

Department of Biochemistry (U38), Faculty of Medicine, University of Porto, Portugal

\begin{abstract}
Angiogenesis can be regarded as a double-edged sword given its useful actions in repairing tissues, and secondly due to its effects in enhancing tissue damage. Angiogenesis is actually involved in the pathogenesis of disorders in which the inflammatory component is a major contributor for disease progression, such as diabetes. It is now stated that angiogenesis together with vasculogenesis partake to neovascular development. A peculiar characteristic of diabetes is the co-existence of vascular impairment and excessive angiogenesis in distinct organs. But despite this knowledge, many questions remain unanswered. Due to the importance of these systems in diabetes-associated complications, a better understanding of the molecular and cellular processes may open new mechanism-based therapeutic options. Accordingly, the available treatment strategies for diabetes management are not completely efficient, as highlighted by the elevated mortality and morbidity rates associated with this condition. Diabetic nephropathy, which may evolve to end-stage renal failure, and diabetic retinopathy that may progress to blindness are important causes of morbidity. Additionally, chronic nonhealing skin ulceration greatly impairs the quality of life of diabetic patients, resulting often in limb amputation. Cardiovascular events, in turn, are responsible for a significant number of deaths in diabetes. This review aims at highlighting the state-of-the-art in diabetic vascular complications, and comprehends the characterization of neovascular system alterations. The elucidation of the diabetic-associated angiogenic paradox is fundamental for prospecting novel therapeutic interventions.
\end{abstract}

Keywords: Endothelial cells, smooth muscle cells, molecular signalling, type 2 diabetes mellitus, vasculopathy.

\section{DIABETES - THE ANGIOGENIC PARADOX}

Diabetes mellitus (DM) is a common metabolic disease with a high and increasing prevalence worldwide. The estimated number of patients afflicted with diabetes, presently about 170 million people, is expected to increase and reach a pandemic rate of 366 million by the year 2030 [1]. This increasing incidence renders DM a leading cause of morbidity and mortality in the near future. DM causes multiple serious health complications, demanding a thorough clinical management, which renders this disease already a main burden for the National Care Systems. The diabetic condition is characterized by and responsible for alterations in micro- and macrovascular beds, by inducing changes in neovascular mechanisms and impairing vascular homeostasis. However, diabetes is a paradoxal disease from the vascular point of view, since in specific organs there is an increase in neovascularization, as it occurs in the kidney and in the retina, whereas others present a marked inhibition of angiogenesis, as in coronary heart disease and peripheral vascular disease [2]. Despite the increasing awareness of this diabetesassociated angiogenic paradox, the molecular mechanisms driven in the distinct organs remain unclear.

\section{THE ANGIOGENIC PROCESS}

The concept of angiogenesis was first refered as the sprouting of ECs from pre-existing vessels into a previously

*Address correspondence to this author at the Department of Biochemistry (U38), Faculty of Medicine, University of Porto, Al. Prof. Hernâni Monteiro, 4200-319 Porto, Portugal; Tel/Fax: 351 225513624;

E-mail: raqsoa@med.up.pt avascular tissue [3]. More recently, this concept was enlarged to the growth and remodeling process by which an initial vascular system is modified to form a complex branching network, characteristic of the mature vasculature [4]. Angiogenesis is, therefore, a complex multistep process involving extracellular matrix (ECM) degradation, proliferation, survival, migration and morphological changes of endothelial cells (EC) and their anastomosis to assemble into a vascular structure [5]. Upon an angiogenic stimulus, ECM proteases release leads to the localized degradation of the blood vessel basement membrane, enabling thus local ECs to change shape, invade stroma and proliferate, forming tubular structures that coalesce. In order for this complex angiogenic process to function, a huge number of anti- and proangiogenic factors must act in a coordinate and synergistic manner to assemble functional blood vessels [5].

\section{ANGIOGENIC STIMULATING CONDITIONS IN DIABETES}

The pathophysiological mechanisms that characterize diabetes encompass high glycemia, nonenzymatic glycation and lipoxidation end products, chronic inflammation, enhanced reactive oxygen species (ROS) and hyperinsulinemia. Vascular cells are, therefore, exposed to abnormal concentrations of a large amount of molecules in DM patients, which result in imbalanced signaling pathways, in comparison to normal (euglycemic) individuals.

\section{Hypoxia}

Hypoxia is probably the major inducer of angiogenesis both in physiological and in pathological situations. The presence of hypoxic environment triggers cells to up- 
regulate hypoxic-inducible factors- $\alpha$ (HIF $\alpha$ ) subunits, which are well-established transcription factors of a huge number of genes, including angiogenic activators, such as vascular endothelial growth factor (VEGF), stromal-derived factor-1 (SDF-1), platelet-derived growth factor (PDGF) or angiopoietin-2 (Ang2) [5-8]. Therefore, angiogenesis process is dependent on oxygen homeostasia, being this mechanism finely tuned by prolyl and lysyl-hydroxylases that target HIF subunits to ubiquitination and degradation through VHL binding [7]. Besides oxygen availability, HIF can further be activated by the presence of ROS or inflammatory cytokines, two diabetic-associated features. ROS results in inactivation of hydroxylases, HIF $\alpha$ protein synthesis induction by mTOR signaling pathway [9]. Hypoxia is a characteristic feature of inflammation as well. The chronic inflammatory status of diabetes renders, thus, hypoxia a mainstay process affecting angiogenesis. Intradermal injection of plasmids containing a constitutively active form of HIF1 $\alpha$ to Lepr $^{\mathrm{db} / \mathrm{db}}$ mice, an in vivo model of type2 DM, revealed an increase in VEGF, placental growth factor (PlGF), PDGF-B and Ang2 plasma levels and promoted skin wound healing, confirming the relevance of hypoxia in diabetes $[7,10]$.

\section{Chronic Inflammation}

DM is characterized by a chronic inflammatory state with concomitant cytokine and growth factor secretion. Consistently, diabetic patients often present defects in neutrophil chemottactive activity, as well as phagocytic and microbial activities [11-13]. Corroborating these findings physiological increases in glycemia after meals aggravate inflammation process through the activation of $\mathrm{NFKB}$, a pro-inflammatory transcription factor $[14,15]$. A potent interplay between these two conditions is well described in many pathological situations $[15,16]$. In fact, inflammatory-induced release of a huge amount of factors (e.g. IL1, IL6, TNF $\alpha$, CCL5), lead to angiogenic stimulation. Cyclo-oxygenase-2 (COX-2) is an inducible enzyme responsible for the increase in prostaglandin (PGE) biosynthesis during inflammation. COX-2 induces angiogenesis by up-regulating VEGF production [14, 15], substantiating the angiogenic stimulating role of inflammation. In addition, PGE2 stimulates angiogenesis by MTMMP-mediated activity of TGF $\beta$ signaling pathway [17]. On the other hand, angiogenesis contributes to inflammation by providing oxygen and nutrients to inflammatory sites.

\section{Oxidative Stress}

Another pathophysiological condition in diabetes is the presence of oxidative and nitrosative stress. Several lines of evidence indicate that ROS activate signaling pathways that promote angiogenesis $[18,19]$. ROS can be formed in many distinct ways in the human organism. $\mathrm{NAD}(\mathrm{P}) \mathrm{H}$ oxidases (Nox) are present in vascular EC and smooth muscle cells (SMC), and are also a relevant source of ROS formation from molecular oxygen $[19,20]$. In addition, a wide variety of angiogenic stimulators can be up-regulated by Nox [19]. EC further present the endothelial isoform of nitric oxide synthase (eNOS), which further contributes to ROS generation [21]. eNOS catalize the synthesis of nitric oxide (NO), an established scavenger of superoxide anion $\left(\mathrm{O}^{-}\right)$, but also a potent vasodilator and angiogenic stimulator. Recently, NO has been reported to promote ROS production [22, 23]. According to these studies, NO may be reduced by the cyto- chrome c oxidase enzyme complex at the mitochondrial electron transport chain (ETC) in specific redox conditions, preventing, therefore, oxygen reduction to water, which in turn leads to ROS accumulation. In diabetes, ROS is extensively produced, either due to the chronic inflammatory status or metabolic changes associated with this disorder (e.g. glucose and fatty acid elevation in plasma). ROS activate then stresssensitive signaling pathways, which might cause insulin resistance and $\beta$-cell dysfunction, two mandatory conditions of diabetes [24].

\section{Hyperglycemia and AGE Products}

Diabetic patients presenting poor glycemic control exhibit high levels of advanced glycation end products (AGE) both in serum and in tissue [25]. AGE are known to promote tissue fibrosis in organs with end-stage damage [26], a condition associated with ECM expansion. This phenomenon results in extensive prevention of tissue perfusion and consequently ischemia-induced angiogenesis. In addition, AGE activates synthesis of various pro-angiogenic proteins, such as insulin-like growth factor binding protein-related protein2 (IGFPB-rP2) in skin fibroblasts and in kidney mesangial cells $[25,26]$. Angiogenic enhancement has also been attributed to DM-persisting glycation. Glycated albumin is a frequent event, resulting in HIF1 $\alpha$ overexpression, and consequently angiogenesis. Accordingly, AGE level increase are also associated with diabetic retinopathy, in which VEGF is overexpressed [27-29]. Moreover, AGE has been involved in pericyte loss as well, being thus considered a promoter of vascular cell derangement. On the other hand, glycation of angiogenic growth factors such as fibroblast growth factor (FGF), hepatocyte growth factors (HFG), PIGF or PDGF, were reported to induce inactivation of their signaling pathways, which might explain at least partially the reduction in vascular response in many diabetic tissues [30]. These findings are, hence, still highly controversial, implying that further studies are mandatory.

Hyperglycemia is also itself an angiogenic enhancer. High glucose levels stimulate the transcription of many angiogenic genes including transforming growth factor (TGF)$\beta$ in vascular smooth muscle cells [32]. This growth factor may in turn up-regulate the expression of VEGF, which has been described in diabetic nephropathy animals [33-35].

Besides hyperglycemia and its derived AGE components, the lack of insulin response within adipocytes enhances lipolysis by increasing hormone-sensitive activity. This, results in free fatty acid, glycerol and monoacylglycerol moieties release to the plasma. One of these components, 1butirylglycerol, prevents vasodilation in long term conditions by creating ischemia, which in turn triggers angiogenesis [25].

\section{Advanced Lipoxidation End Products (ALE)}

Another group of molecules known to play a role in diabetic vasculopathy is advanced lipoxidation end products (ALE). These are proteins that were irreversibly modified by lipid reactive species, such as carbonyl and malondialdehyde. Recent studies showed that the presence of ALE in diabetic patients strongly associates with chronic inflammation [31]. In agreement, synthetic ALE effectively increases the expression of a wide range of inflammatory factors, such 
as CXCL10, CCL2, COX-2, $\beta 1$ and $\beta 2$ integrins, IL6, IL8 and inducible NOS (iNOS) in monocytes [31]. Most of these monocytic dysfunction molecules are established angiogenic activators, implying that ALE products are also likely to present pro-angiogenic properties.

\section{MECHANISMS OF ANGIOGENESIS IN DIABETES}

Angiogenesis requires the activation of many receptors by specific ligands, such as PIGF, acidic and basic fibroblast growth factors (aFGF and bFGF, respectively), angiopoietins, HGF, PDGF, and platelet derived-endothelial cell growth factor (PD-ECGF), among many others [36-40]. Despite the increasing number of factors playing a role in angiogenesis, VEGF signalling on ECs represents the major ratelimiting step during the process [41]. VEGF engages and activates its tyrosine kinase receptors VEGFR-1 and VEGFR-2 on ECs. Despite VEGF binding to both receptors, most of its biological functions are mediated by VEGFR-2 signaling, which is involved in several steps of the angiogenic process, leading to the assembly of a new vasculature [42-44]. In addition, VEGF is also the major factor involved in EPCs mobilization from the bone marrow to the peripheral circulation and to angiogenic sites, where they differentiate and integrate the neovasculature [45, 46] (please also see paper on EPCs in this issue).

Most of these receptors present in vascular wall cells act by triggering sinaling cascades of phosphorylating kinases. Nonetheless, for these cellular transduction pathways to properly function, a balance between phosphorylating kinases and dephosphorylating phosphatases must exist. Protein tyrosine phosphatases (PTP) are a very large family of enzymes that catalize dephosphorylation of tyrosine residues [47]. Diabetes-related insults including hyperglycemia, oxidative stress and insulin resistance act frequently in concert in vascular cells imbalancing phosphorylation/dephosphorylation reactions, which results often in enhancement of protein phosphorylation. Vessel remodeling is a consequence of PTP phosphorylation. VCAM-1-dependent lymphocyte extravasation through the endothelial layer requires PTP1B phosphorylation, a non-receptor PTP largely expressed that presents a variety of regulating properties [48]. Interestingly enough, inhibition of PTP1B enhances EGFR phosphorylation and activity, which results in proliferation in corneal EC [49]. Therefore, diabetes increases PTP1B activity in retina, preventing autophosphorylation of retinal insulin receptor [50]. Altogether, these finding emphasize the relevance of this family of phosphatases in diabetes-derived cvascular complications, by their direct actions within angiogenic phosphorylating signaling pathways. Targeting PTP may be a putative strategy for improving insulin sensitivity in diabetic patients.

\section{ANGIOGENESIS AND DIABETIC RETINOPATHY}

Proliferative diabetic retinopathy (DR) is a progressive disorder characterized by the presence of retinal vessel microaneurisms, hemorrhages, exudates and edema [51] (please see paper on DR on this issue). One of the primary changes in the vascular process of DR is the loss of pericytes in retinal capillaries, which may lead to vascular failure and chronic hypoxia. HIF transcription factors, then, promote the rapid formation of neovessels, ultimately resulting in exacerbated angiogenesis $[8,15,29,52,53]$. The sudden estab- lishment of angiogenic vessels leads to the assembly of leaky and malfunctioning vascular structures, which is further enhanced by the presence of a delicate basement membrane [54]. VEGF is released by several distinct cells within the eye. And recent studies showed that VEGFR2 is also overexpressed in retinas from diabetic rats [55]. Diabetic patients exhibiting DR or macular edema present high levels of VEGF [56]. Despite VEGF is the most well established marker of proliferative DR, other factors also play a role. Pigment epithelium-derived factor (PEDF) is an endogenous angiogenic inhibitor factor with reduced levels in several ocular diseases. This factor is known to prevent VEGF and erythropoietin in retinal endothelia cells [57]. Interestingly, normal levels of PEDF were found in the vitreous fluid of diabetic patients. However, a higher molecular-weight isoform was observed in a subgroup of these patients in comparison to non diabetic controls [58]. The same study also revealed that thrombospondin-1 (TSP1) another antiangiogenic factor was reduced in the patients exhibiting the PEDF higher molecular weight isoform [58], implying that a cross talk between these two angiogenic inhibiting factors is likely to occur. TSP1 binds to CD36, a membrane glycoprotein present in many types of cells including microvascular endothelial cells [59]. Remarkably, CD36 also binds to long chain fatty acids and promotes their transport into cells [59]. The fact that CD36 contributes to lipid absorption and metabolism (and also in inflammatory response and oxidative stress) emphasizes its putative implication in metabolic disorders such as diabetes. In addition, hyperglycemia enhances the overexpression of fibronectin, a glycoprotein present in ECM. Increased fibronectin enhances the availability of fibronectin fragments that present pro-angiogenic properties [60]. These fragments may therefore account for the aberrant angiogenesis in retinopathy.

In conclusion, many angiogenic factors play a role in diabetic retinopathy. VEGF is, by far, the most well described, being thus antiVEGF agent an exciting therapeutic strategy. Nevertheless, the complexity of this disorder requires the elucidation of the other molecular mechanisms, in order to search for further therapeutic options.

\section{ANGIOGENESIS AND DIABETIC NEPHROPATHY}

The incidence of end-stage kidney disease is significantly increasing in the western world [61], and this has been attributed to the increasing prevalence of diabetes. Indeed, nephropathy, which is associated with decrease in glomerular filtration rate and increasing creatinine serum levels, emerges 10-15 years after the onset of diabetes [25, 61, 62]. Glomeruli capillaries area is known to increase in STZ diabetic rats when compared to normal age-matched controls [61], implying a pronounced angiogenic reaction to the diabetic condition. VEGF has long been established as a permeability factor $[3,4,5,15,16]$. In kidney, podocytes are able to secrete VEGF, which in turn takes part in the capillary hyperpermeability of macromolecules in glomeruli [62]. VEGF is overexpressed in diabetic kidneys and its role as a neurotrophic and neurogenic agent has recently been reported [63]. Recently, blockade of VEGF signalling pathway resulted in attenuation of diabetic albuminuria [62]. Diabetic animals exhibit increased expression of extracellular matrix components in renal cortex and glumeruli [34]. Another relevant angiogenic markers present in diabetic nephropathy 
is transforming growth factor $\beta$ (TGF $\beta$ ) and its type II receptor $[34,35]$. This factor is further markedly excreted in the urine in diabetic patients [35]. Altogether these studies emphasize the angiogenic enhancement that is characteristic of diabetic patients.

\section{ANGIOGENESIS AND WOUND HEALING}

One of the major drawbacks of diabetes is the development of chronic, nonhealing foot ulcerations, which significantly impairs life quality of these patients and results often in lower-limb amputation in the worst cases [64]. Wound healing is a complex process involving a wide variety of cells and growth factors. Normal wound healing requires the involvement of many cell types and cytokines. Platelets that are recruited upon skin injury, together with keratinocytes and inflammatory cells such as macrophages, release several angiogenic factors including PDGF, VEGF, TGF $\beta$ and bFGF [64-66]. The excessive PDGF produced leads to recruitment of fibroblasts to the injured tissue, which in turn supply new ECM components to the damaged area [66].

Nevertheless, the interplay between the various stimulating agents present in diabetes, namely oxidative stress, hyperglycemia and related AGE products, inflammation and neuropathy, a long-term complication of diabetic characterized by a progressive loss of nerve fibres, contribute enormously to the impairment of diabetic wound healing [67]. Fibroblasts from diabetic mice release reduced levels of VEGF release when compared to wild type animals [68]. Remarkably, fibroblasts-derived VEGF does not seem to be induced by hypoxia in diabetes [68]. Consequently, reduced levels of VEGF result in impaired recruitment of many cell types to the injured tissue, as well as in decreased (lymph)angiogenesis, and vasculogenesis [69]. The broad effects exerted by VEGF both in vascular, inflammatory and neurogenic processes render this factor a putative therapeutic agent. Preclinical studies in diabetic models using VEGF gene therapy improved blood flow and neurovascular function in diabetic wounds [70]. In agreement, VEGF gene transfer approaches are currently being established in ischemic and neurodegenerative pathological conditions. In summary, PDGF and VEGF seem to play a pivotal role in wound healing. The fact that these two factors are significantly down-regulated in diabetic wounds makes them promising targets for therapeutic purposes.

\section{THERAPEUTIC STRATEGIES TARGETING ANGI- OGENESIS}

The development and progression of diabetes is associated with a large number of complications, which comprise glucose intolerance, insulin resistance, visceral obesity, hypertension and dyslipidemia, reaching epidemic proportions nowadays, and, consequently, presenting a large economic impact on healthcare providers. Many attempts have been made regarding the development of therapeutic strategies for DM. These include incretin agonists (e.g. glucagon-like peptide-1 analogues), cannabinoid receptor antagonists and PPAR and insulin agonists [16, 71]. Nonetheless, the complex etiopathogenesis of this condition that gathers together genetic factors and life-style features makes this disease a major public health problem. Recent advances on the vascular biology pathways complying diabetes provides clues for the development of new pharmacological mole- cules. The strong implication of VEGF in vasculoproliferative diabetic retinopathy led to the intravitreal use of antiVEGF agents in the management of these patients. Accordingly, three VEGF antagonist pharmacological agents: pegaptanib (Macugen), ranibizumab (lucentis) and bevacizumab (avastin) are currently available for DR treatment [72]. The first two are actually approved for age-related macular degeneration by the Food and Drug Administration (FDA), and clinical trials are ongoing for their clinical use in other ocular vasculoproliferative disorders including DR (see paper on diabetic retinopathy in this issue). Yet, the coexistence of aberrant vascularization (occuring in retinopathy and nephropathy) together with angiogenesis impairment (such as in peripheral vascular disease and diabeticassociated vascular disease), provides angiogenic systemic therapy a dangerous option for diabetes. Accordingly, although inhibition of VEGF could be very useful in controling diabetic retinopathy, it might trigger undesired sideeffects including impaired wound healing, abrogation of collateral vessel growth, hypertension and proteinuria, which are prevalent features of diabetic patients. Therefore, a careful evaluation of the molecular vascular events and their association with other diabetic processes (e.g. chronic oxidative stress, inflammation, etc) must be accomplished before addressing these pathways towards treatment.

Wound healing is a major complication of diabetes with extensive morbidity rates where angiogenesis is effectively impaired. Vascularization in wound healing can be promoted by several interventions. These include therapeutic agents involving growth factors, bioactive matrices, mechanical systems, tissue engineering biomaterials and hyperbaric oxygen therapy (HBO) $[16,73]$. HBO for instances is already in use in the clinic, and stimulates wound healing by promoting growth factor synthesis at the wound bed, and by mobilizing EPCs [74, 75]. HBO improves microvascular perfusion, probably by stimulating NO synthesis through the hyperbaric oxygen enhancement. In addition, it also helps reducing tissue edema and improves leukocyte oxygendependent phagocytic capacity. HBO is indicated in hypoxic ulcers in diabetic patients that do not present contraindications such as retinopathy, emphysema or cancer.

Due to their health promoting properties, dietary-derived polyphenols attain to the various pathophysiological conditions associated with diabetes [76, 77]. In fact, increasing epidemiological and experimental evidence indicates that these compounds are major candidates for treatment or prevention of diabetes and many other human disorders that combine oxidative stress, chronic inflammation and imbalanced vascular patterns. Besides, these compounds attract a lot of attention due to their low cost, easy availability and low toxic effects. Despite a huge number of studies are in progress, several unsolved questions must be taken into account before these molecules can be recommended as preventive or therapeutic agents, namely, their bioavailability, dose, specificity, feasibility, distribution, degradation and elimination from our body.

An increasing interest has been lately raised on the use of nicotine as an angiogenic promoter [66, 78]. Nicotine is considered as a potential therapeutic agent in neurodegenerative disease or ulcerative colitis [79, 80]. Nicotine binds to N-nACh receptors. In the endothelium, this 
natural alkaloid binds $\alpha 7 \mathrm{~N}$-nACh receptor, inducing cell proliferation and migration, two crucial steps of the angiogenic process. Although diabetic patients who smoke experience impaired wound healing processes and accelerate atherosclerosis and the development of several types of cancer, the topic use of nicotine (transdermal patches) in skin wounds seems to be advantageous in certain conditions [66, 78]. Again, dosing and time course studies are mandatory before nicotine synthetic analogs can be used in the clinic.

In conclusion, vascular complications are key players in diabetes. However, many questions still remain regarding the use of angiogenesis as a therapeutic approach. Translation and clinical research are critical in the near future to elucidate the inherent molecular mechanisms that hold the angiogenic paradox, and to predict which patients could benefit from each therapeutic approach.

\section{REFERENCES}

[1] Wild, S.; Roglic, G.; Green, A.; Sicree, R.; King, H. Global prevalence of diabetes: estimates for the year 2000 and projections for 2030. Diabetes Care, 2004, 27, 1047-1053.

[2] Stratton, I.M.; Adler, A.I.; Neil, H.A.; Matthews, D.R.; Manley, S.E.; Cull, C.A.; Hadden, D.; Turner, R.C.; Holman, R.R. Association of glycaemia with macrovascular and microvascular complications of type 2 diabetes (UKPDS 35): prospective observational study. Br. Med. J., 2000, 321, 405-12.

[3] Folkman, J.; Shing, Y. Angiogenesis. J. Biol. Chem., 1992, 267, 10931-4.

[4] Carmeliet, P. Angiogenesis in life, disease and medicine. Nature, 2005, 438, 932-6.

[5] Carmeliet, P.; Jain, R.K. Angiogenesis in cancer and other diseases. Nature, 2000, 407, 249-57.

[6] Semenza, G.L.; Shimoda, L.A.; Prabhakar, N.R. Regulation of gene expression by HIF-1. Novartis Found. Symp., 2006, 272, 2-8.

[7] Semenza, G.L. Regulation of vascularization by hypoxia-inducible factor 1. Ann. N. Y. Acad. Sci., 2009, 1177, 2-8.

[8] Soares, R.; Costa, C. Angiogenesis and inflammatory diseases: current concepts and therapeutic perspectives. In: Angiogenesis. Basic Science and Clinical Applications, Maragoudakis, M.E.; Papadimitriou, E., Eds.; Transworld Research Network: Kerala, 2007, pp. 511-47.

[9] Yuan, G.; Nanduri, J.; Khan, S.; Semenza, G.L.; Prabhakar, N.R. Induction of HIF-1alpha expression by intermittent hypoxia: involvement of NADPH oxidase, $\mathrm{Ca} 2+$ signaling, prolyl hydroxylases, and mTOR. J. Cell. Physiol., 2008, 217, 674-85.

[10] Liu, L.; Marti, G.P.; Wei, X.; Zhang, X.; Zhang, H.; Liu, Y.V.; Nastai, M.; Semenza, G.L.; Harmon, J.W. Age-dependent impairment of HIF-1alpha expression in diabetic mice: Correction with electroporation-facilitated gene therapy increases wound healing, angiogenesis, and circulating angiogenic cells. J. Cell. Physiol., 2008, 217, 319-27.

[11] Alba-Loureiro, T.C.; Hirabara, S.M.; Mendonça, J.R.; Curi, R.; Pithon-Curi, T.C. Diabetes causes marked changes in function and metabolism of rat neutrophils. J. Endocrinol., 2006, 188, 295-303.

[12] Mastej, K.; Adamiec, R. Neutrophil surface expression of CD11b and CD62L in diabetic microangiopathy. Acta Diabetol., 2008, 45, 183-90.

[13] Marhoffer, W.; Stein, M.; Schleinkofer, L.; Federlin, K. Evidence of ex vivo and in vitro impaired neutrophil oxidative burst and phagocytic capacity in type 1 diabetes mellitus. Diabetes Res. Clin. Pract., 1993, 19, 183-8.

[14] Dickinson, S.; Hancock, D.P.; Petocz, P.; Ceriello, A.; BrandMiller, J. High-glycemic index carbohydrate increases nuclear factor-kappaB activation in mononuclear cells of young, lean healthy subjects. Am. J. Clin. Nutr., 2008, 87, 1188-93.

[15] Costa, C.; Incio, J.; Soares, R. Angiogenesis and chronic inflammation: cause or consequence? Angiogenesis, 2007, 10, 149-66.

[16] Soares, R. Angiogenesis in metabolic syndrome. In: Oxidative Stress, Inflammation and Angiogenesis in Metabolic Syndrome,
Soares, R.; Costa, C. Eds.; Springer-Verlag: The Netherlands, 2009; pp 85-99.

[17] Alfranca, A.; López-Oliva, J.M.; Genís, L.; López-Maderuelo, D.; Mirones, I.; Salvado, D.; Quesada, A.J.; Arroyo, A.G.; Redondo, J.M. PGE2 induces angiogenesis via MT1-MMP-mediated activation of the TGFbeta/Alk5 signaling pathway. Blood, 2008, 112, 1120-8.

[18] Thannickal, V.J.; Fanburg, B.L. Reactive oxygen species in cell signaling. Am. J. Physiol. Lung Cell. Mol. Physiol., 2000, 279, L1005-28.

[19] Polytarchou, C.; Hatziapostolou, M.; Poimenidi, E.; Papadimitriou, E. Reactive oxygen species as mediators of angiogenesis signaling. In: Angiogenesis. Basic Science and Clinical Applications, Maragoudakis, M.E.; Papadimitriou, E., Eds.; Transworld Research Network: Kerala, 2007, pp. 207-227.

[20] Blanchetot, C.; Boonstra, J. The ROS-NOX connection in cancer and angiogenesis. Crit. Rev. Eukaryot. Gene Exp., 2008, 18, 35-45.

[21] Vásquez-Vivar, J.; Kalyanaraman, B.; Martásek, P.; Hogg, N.; Masters, B.S.; Karoui, H.; Tordo, P.; Pritchard, K.A. Jr. Superoxide generation by endothelial nitric oxide synthase: the influence of cofactors. Proc. Natl. Acad. Sci. USA, 1998, 95, 9220-5.

[22] Palacios-Callender, M.; Hollis, V.; Mitchison, M.; Frakich, N.; Unitt, D.; Moncada, S. Cytochrome c oxidase regulates endogenous nitric oxide availability in respiring cells: a possible explanation for hypoxic vasodilation. Proc. Natl. Acad. Sci. USA., 2007, 104, 18508-13.

[23] Erusalimsky, J.D.; Moncada, S. Nitric oxide and mitochondrial signaling: from physiology to pathophysiology. Arterioscler. Thromb. Vasc. Biol., 2007, 27, 2524-31.

[24] Calhau, C.; Santos, A. Oxidative stress in the metabolic syndrome. In: Oxidative Stress, Inflammation and Angiogenesis in Metabolic Syndrome, Soares, R.; Costa, C, Eds.; Springer-Verlag: The Netherlands, 2009, pp. 33-63.

[25] Martin, A.; Komada, M.R.; Sane, D.C. Abnormal angiogenesis in diabetes mellitus. Med. Res. Rev., 2003, 23, 117-45.

[26] Twigg, S.M.; Chen, M.M.; Joly, A.H.; Chakrapani, S.D.; Tsubaki, J.; Kim, H.S.; Oh, Y.; Rosenfeld, R.G. Advanced glycosylation end products up-regulate connective tissue growth factor (insulin-like growth factor-binding protein-related protein 2) in human fibroblasts: a potential mechanism for expansion of extracellular matrix in diabetes mellitus. Endocrinology, 2001, 142, 1760-9.

[27] Fonseca, V.; Munshi, M.; Merin, L.M.; Bradford, J.D. Diabetic retinopathy: a review for the primary care physician. South Med. J., 1996, 89, 839-50.

[28] Singh, R.; Barden, A.; Mori, T.; Beilin, L. Advanced glycation endproducts: a review. Diabetologia, 2001, 44, 129-46.

[29] Aiello, L.P.; Avery, R.L.; Arrigg, P.G.; Keyt, B.A.; Jampel, H.D.; Shah, S.T.; Pasquale, L.R.; Thieme, H.; Iwamoto, M.A.; Park, J.E.; et al. Vascular endothelial growth factor in ocular fluid of patients with diabetic retinopathy and other retinal disorders. N. Engl. J. Med., 1994, 331, 1480-7.

[30] Schmidt, A.M.; Yan, S.D.; Wautier. J.L.; Stern, D. Activation of receptor for advanced glycation end products: a mechanism for chronic vascular dysfunction in diabetic vasculopathy and atherosclerosis. Circ. Res., 1999, 84, 489-97.

[31] Shanmugam, N.; Figarola, J.L.; Li, Y.; Swiderski, P.M.; Rahbar, S.; Natarajan, R. Proinflammatory effects of advanced lipoxidation end products in monocytes. Diabetes, 2008, 57, 879-88.

[32] Stark, J.; Baffour, R.; Garb, J.L.; Kaufman, J.; Berman, J.; Rhee, S.; Norris, M.A.; Friedmann, P. Basic fibroblast growth factor stimulates angiogenesis in the hindlimb of hyperglycemic rats. $J$. Surg. Res., 1998, 79, 8-12.

[33] Han, D.C.; Hoffman, B.B.; Hong, S.W.; Guo, J.; Ziyadeh, F.N. Therapy with antisense TGF-betal oligodeoxynucleotides reduces kidney weight and matrix mRNAs in diabetic mice. Am. J. Physiol. Renal Physiol., 2000, 278, F628-34.

[34] Ziyadeh, F.N.; Hoffman, B.B.; Han, D.C.; Iglesias-De La Cruz, M.C.; Hong, S.W.; Isono, M.; Chen, S.; McGowan, T.A.; Sharma, $\mathrm{K}$. Long-term prevention of renal insufficiency, excess matrix gene expression, and glomerular mesangial matrix expansion by treatment with monoclonal antitransforming growth factor-beta antibody in db/db diabetic mice. Proc. Natl. Acad. Sci. USA., 2000, 97, 8015-20. 
[35] Sharma, K.; Ziyadeh, F.N. Hyperglycemia and diabetic kidney disease. The case for transforming growth factor-beta as a key mediator. Diabetes, 1995, 44, 1139-46.

[36] Yancopoulos, G.D.; Davis, S.; Gale, N.W.; Rudge, J.S.; Wiegand, S.J.; Holash, J. Vascular-specific growth factors and blood vessel formation. Nature, 2000, 407, 242-248.

[37] Presta, M.; Dell'Era, P.; Mitola, S.; Moroni, E.; Ronca, R.; Rusnati, M. Fibroblast growth factor/fibroblast growth factor receptor system in angiogenesis. Cytokine Growth Factor Rev., 2000, 16, 15978.

[38] Miyazono, K.; Usuki, K.; Heldin, C.H. Platelet-derived endothelial cell growth factor. Prog. Growth Factor Res., 1991, 3, 207-17.

[39] Ohnishi, T.; Daikuhara, Y. Hepatocyte growth factor/scatter factor in development, inflammation and carcinogenesis: its expression and role in oral tissues. Arch. Oral Biol., 2003, 48, 797-04.

[40] Luttun, A.; Tjwa, M.; Carmeliet, P. Placental growth factor (PlGF) and its receptor Flt-1 (VEGFR-1): novel therapeutic targets for angiogenic disorders. Ann. N. Y. Acad. Sci., 2002, 979, 80-93.

[41] Ferrara, N.; Gerber, H.P.; LeCouter, J. The biology of VEGF and its receptors. Nat. Med., 2003, 9, 669-76.

[42] Wheeler-Jones, C.; Abu-Ghazaleh, R.; Cospedal, R. Houliston, R.A.; Martin, J.; Zachary, I. Vascular endothelial growth factor stimulates prostacyclin production and activation of cytosolic phospholipase A2 in endothelial cells via p42/p44 mitogenactivated protein kinase. FEBS Lett., 1997, 420, 28-32.

[43] Gerber, H.P.; McMurtrey, A.; Kowalski, J.; Yan, M.; Keyt, B.A.; Dixit, V.; Ferrara, N. Vascular endothelial growth factor regulates endothelial cell survival through the phosphatidylinositol 3'kinase/Akt signal transduction pathway. Requirement for Flk1/KDR activation. J. Biol. Chem., 1998, 273, 30336-43.

[44] Gerber, H.P.; Dixit, V.; Ferrara, N. Vascular endothelial growth factor induces expression of the antiapoptotic proteins $\mathrm{Bcl}-2$ and A1 in vascular endothelial cells. J. Biol. Chem., 1998, 273, 133136.

[45] Asahara, T.; Takahashi, T.; Masuda, H.; Kalka, C.; Chen, D.; Iwaguro, H.; Inai, Y.; Silver, M.; Isner, J.M. VEGF contributes to postnatal neovascularization by mobilizing bone marrow-derived endothelial progenitor cells. EMBO J., 1999, 18, 3964-72.

[46] Costa, C.; Soares, R.; Schmitt, F. Angiogenesis: now and then. APMIS, 2004, 112, 402-12.

[47] Popov, D. Vascular PTPs: current developments and challenges for exploitation in Type 2 diabetes-associated vascular dysfunction. Biochem. Biophys. Res. Commun., 2009, 389, 1-4.

[48] Deem, T.L.; Abdala-Valencia, H.; Cook-Mills, J.M. VCAM -1 activation of endothelial cell protein tyrosine phosphatase $1 \mathrm{~B}$. $J$. Immunol., 2007, 178, 3865-73.

[49] Harris, D.L.; Joyce, N.C. Protein tyrosine phosphatase, PTP1B, expression and activity in rat corneal endothelial cells. Mol. Vis., 2007, 13, 785-96.

[50] Rajala, R.V.; Wiskur, B.; Tanito, M.; Callegan, M.; Rajala, A. Diabetes reduces autophosphorylation of retinal insulin receptor and increases protein-tyrosine phosphatase-1B activity. Invest. Ophthalmol. Vis. Sci., 2009, 50, 1033-40.

[51] Aksoy, H.; Akçay, F.; Kurtul, N.; Baykal, O.; Avci, B. Serum 1,25 dihydroxy vitamin D $(1,25(\mathrm{OH}) 2 \mathrm{D} 3), 25$ hydroxy vitamin D $(25(\mathrm{OH}) \mathrm{D})$ and parathormone levels in diabetic retinopathy. Clin. Biochem., 2000, 33, 47-51.

[52] Lip, P.L.; Chatterjee, S.; Caine, G.J.; Hope-Ross, M.; Gibson, J.; Blann, A.D.; Lip, G.Y. Plasma vascular endothelial growth factor, angiopoietin-2, and soluble angiopoietin receptor tie-2 in diabetic retinopathy: effects of laser photocoagulation and angiotensin receptor blockade. Br. J. Ophthalmol., 2004, 88, 1543-6.

[53] Rand, L.I. Recent advances in diabetic retinopathy. Am. J. Med., 1981, 70, 595-602.

[54] Schultz, G.S.; Grant, M.B. Neovascular growth factors. Eye, 1991, 5, 170-80.

[55] Chou, E.; Suzuma, I.; Way, K.J.; Opland, D.; Clermont, A.C.; Naruse, K.; Suzuma, K.; Bowling, N.L.; Vlahos, C.J.; Aiello, L.P.; King, G.L. Decreased cardiac expression of vascular endothelial growth factor and its receptors in insulin-resistant and diabetic States: a possible explanation for impaired collateral formation in cardiac tissue. Circulation, 2002, 105, 373-9.
[56] Duh, E.; Aiello, L.P. Vascular endothelial growth factor and diabetes: the agonist versus antagonist paradox. Diabetes, 1999, 48, 1899-906.

[57] Elayappan, B.; Ravinarayannan, H.; Sardar-Pasha, S.P.; Lee, K.J.; Gurunathan, S. PEDF inhibits VEGF- and EPO- induced angiogenesis in retinal endothelial cells through interruption of PI3K/Akt phosphorylation. Angiogenesis, 2009, 12, 313-24.

[58] Wang, M.; Wang, J.J.; Li, J.; Park, K.; Qian, X.; Ma, J.X.; Zhang, S.X. Pigment Epithelium-derived factor (PEDF) suppresses adipogenesis via inhibition of the MAPK/ERK pathway in 3T3-L1 preadipocytes. Am. J. Physiol. Endocrinol. Metab., 2009, 297, E1378-87.

[59] Silverstein, R.L.; Febbraio, M. CD36, a scavenger receptor involved in immunity, metabolism, angiogenesis, and behavior. Sci. Signal., 2009, 2, re3.

[60] Grant, M.B.; Caballero, S.; Bush, D.M.; Spoerri, P.E. Fibronectin fragments modulate human retinal capillary cell proliferation and migration. Diabetes, 1998, 47, 1335-40.

[61] Nyengaard, J.R.; Rasch, R. The impact of experimental diabetes mellitus in rats on glomerular capillary number and sizes. Diabetologia, 1993, 36, 189-94.

[62] Chen, S.; Ziyadeh, F.N. Vascular endothelial growth factor and diabetic nephropathy. Curr. Diab. Rep., 2008, 8, 470-6.

[63] Raab, S.; Plate, K.H. Different networks, common growth factors: shared growth factors and receptors of the vascular and the nervous system. Acta Neuropathol., 2007, 113, 607-26.

[64] Pradhan, L.; Nabzdyk, C.; Andersen, N.D.; LoGerfo, F.W.; Veves, A. Inflammation and neuropeptides: the connection in diabetic wound healing. Expert Rev. Mol. Med., 2009, 11, e2.

[65] Bao, P.; Kodra, A.; Tomic-Canic, M.; Golinko, M.S.; Ehrlich, H.P.; Brem, H.The role of vascular endothelial growth factor in wound healing. J. Surg. Res., 2009, 153, 347-58.

[66] Martin, J.W.; Mousa, S.S.; Shaker, O.; Mousa, S.A. The multiple faces of nicotine and its implications in tissue and wound repair. Exp. Dermatol., 2009, 18, 497-505.

[67] Urbancic-Rovan, V. Causes of diabetic foot lesions. Lancet, $\mathbf{2 0 0 5}$ 366, 1675-6.

[68] Lerman, O.Z.; Galiano, R.D.; Armour, M.; Levine, J.P.; Gurtner, G.C. Cellular dysfunction in the diabetic fibroblast: impairment in migration, vascular endothelial growth factor production, and response to hypoxia. Am. J. Pathol., 2003, 162, 303-12.

[69] Asai, J.; Takenaka, H.; Katoh, N.; Kishimoto, S. Dibutyryl cAMP influences endothelial progenitor cell recruitment during wound neovascularization. J. Invest. Dermatol., 2006, 126, 1159-67.

[70] Carmeliet, P.; Storkebaum, E. Vascular and neuronal effects of VEGF in the nervous system: implications for neurological disorders. Semin. Cell. Dev. Biol., 2002, 13, 39-53.

[71] Lebovitz, H.E. Therapeutic options in development for management of diabetes: pharmacologic agents and new technologies. Endocr. Pract., 2006, 12(Suppl 1), 142-7.

[72] Jardeleza, M.S.; Miller, J.W. Review of anti-VEGF therapy in proliferative diabetic retinopathy. Semin. Ophthalmol., 2009, 24, 87-92.

[73] Li, W.W.; Hutnik, M.; Li, V. Angiogenesis-based medicine: principles and practice for disease prevention and intervention. In: Angiogenesis. Basic Science and Clinical Applications, Maragoudakis, M.E.; Papadimitriou, E., Eds.; Transworld Research Network. Kerala, 2007, pp. 377-417.

[74] Gallagher, K.A.; Goldstein, L.J.; Thom, S.R.; Velazquez, O.C. Hyperbaric oxygen and bone marrow-derived endothelial progenitor cells in diabetic wound healing. Vascular, 2006, 14, 328-37.

[75] Goldstein, L.J.; Gallagher, K.A.; Bauer, S.M.; Bauer, R.J.; Baireddy, V.; Liu, Z.J.; Buerk, D.G.; Thom, S.R.; Velazquez, O.C. Endothelial progenitor cell release into circulation is triggered by hyperoxia-induced increases in bone marrow nitric oxide. Stem Cells, 2006, 24, 2309-18.

[76] Negrão, R.; Faria, A. Natural polyphenols as anti-oxidant, antiinflammatory and anti-angiogenic agents in the metabolic syndrome. In: Oxidative Stress, Inflammation and Angiogenesis in Metabolic Syndrome, Soares, R.; Costa, C., Eds.; Springer-Verlag: The Netherlands, 2009, pp .147-179.

[77] Van Cleemput, M.; Cattoor, K.; De Bosscher, K.; Haegeman, G.; De Keukeleire, D.; Heyerick, A. Hop (Humulus lupulus)-derived 
bitter acids as multipotent bioactive compounds. J. Nat. Prod., 2009, 72, 1220-30.

[78] Costa, F.; Soares, R. Nicotine: a pro-angiogenic factor. Life Sci., 2009, 84, 785-90.

[79] Chan, A.L.; Chien, Y.W.; Jin-Lin, S. Transdermal delivery of treatment for Alzheimer's disease: development, clinical performance and future prospects. Drugs Aging, 2008, 25, 761-75.
[80] Takeuchi, H.; Yanagida, T.; Inden, M.; Takata, K.; Kitamura, Y.; Yamakawa, K.; Sawada, H.; Izumi, Y.; Yamamoto, N.; Kihara, T.; Uemura, K.; Inoue, H.; Taniguchi, T.; Akaike, A.; Takahashi, R.; Shimohama, S. Nicotinic receptor stimulation protects nigral dopaminergic neurons in rotenone-induced Parkinson's disease models. J. Neurosci. Res., 2009, 87, 576-85.

(C) Raquel Soares; Licensee Bentham Open.

This is an open access article licensed under the terms of the Creative Commons Attribution Non-Commercial License (http://creativecommons.org/licenses/by-nc/3.0/) which permits unrestricted, non-commercial use, distribution and reproduction in any medium, provided the work is properly cited. 